\title{
Dclk1 in tuft cells promotes inflammation-driven epithelial restitution and mitigates chronic colitis
}

\author{
Jun $\mathrm{Yi}^{1,2} \cdot$ Kirk Bergstrom ${ }^{2} \cdot$ Jianxin Fu $\mathbb{1}^{2} \cdot$ Xindi Shan ${ }^{2} \cdot$ J. Michael McDaniel ${ }^{2} \cdot$ Samuel McGee ${ }^{2}$ Dongfeng $\mathrm{Qu}^{3}$. \\ Courtney W. Houchen ${ }^{3} \cdot$ Xiaowei Liu ${ }^{1} \cdot$ Lijun Xia $\mathbb{1}^{2,4}$
}

Received: 1 May 2018 / Revised: 28 September 2018 / Accepted: 22 October 2018 / Published online: 26 November 2018

(c) ADMC Associazione Differenziamento e Morte Cellulare 2018

\begin{abstract}
Ulcerative colitis (UC) is a chronic inflammatory bowel disease characterized by defective intestinal barrier integrity toward the microbiota and epithelial damage. Double cortin-like kinase 1 (Dclk1), a marker of intestinal tuft cells, can regulate tissue regenerative responses, but its role in epithelial repair during bacterial-dependent chronic colitis is unclear. We addressed this question using our recently developed mouse model of spontaneous microbiota-dependent colitis induced by mucin-type $O$-glycan deficiency (DKO), which recapitulates most features of human UC. We generated DKO mice lacking intestinal epithelial Dclkl (DKO;Dclkl ${ }^{\Delta I E C}$ ) and analyzed colitis onset and severity using clinical and histologic indices, immune responses by qPCR and immunostaining, and epithelial responses using proliferation markers and organoid culture. We found 3-4-week-old DKO;Dclk1 ${ }^{\Delta I E C}$ mice developed worsened spontaneous colitis characterized by reduced body weight, loose stool, severe colon thickening, epithelial lesions, and inflammatory cell infiltrates compared with DKO mice. The primary defect was an impaired epithelial proliferative response during inflammation. Dclk1 deficiency also reduced inflammation-induced proliferation and growth of colon organoids ex vivo. Mechanistically, Dclk1 expression was important for inflammation-induced Cox2 expression and prostaglandin E2 (PGE2) production in vivo, and PGE2 rescued proliferative defects in Dclk1-deficient colonic organoids. Although tuft cells were expanded in both DKO and DKO; $D c l k l^{\Delta I E C}$ relative to WT mice, loss of Dclk1 was associated with reduced tuft cell activation (i.e., proliferation) during inflammation. Similar results were found in DKO vs. DKO;Dclk $1^{\Delta I E C}$ mice at 3-6 months of age. Our results support that tuft cells, via Dclk1, are important responders to bacterial-induced colitis by enhancing epithelial repair responses, which in turn limits bacterial infiltration into the mucosa.
\end{abstract}

These authors contributed equally: Jun Yi, Kirk Bergstrom.

Edited by Y Shi.

Electronic supplementary material The online version of this article (https://doi.org/10.1038/s41418-018-0237-x) contains supplementary material, which is available to authorized users.

Xiaowei Liu

liuxw@csu.edu.cn

$\triangle$ Lijun Xia

Lijun-Xia@omrf.org

1 Department of Gastroenterology, Xiangya Hospital of Central South University, Changsha, Hunan 410008, China

\section{Introduction}

Ulcerative colitis (UC) is a type of inflammatory bowel disease (IBD) [1], characterized by spontaneous chronic, relapsing-remitting inflammation in the colon, typically presenting as bloody diarrhea and chronic pain [2]. Aberrant interactions of the colonic mucosa with resident microbiota in genetically susceptible individuals are central for disease pathogenesis [3,4]. The result is mucosal ulceration due to inflammation-induced epithelial erosion and death,

2 Cardiovascular Biology Research Program, Oklahoma Medical Research Foundation, Oklahoma City, OK 73104, USA

3 Department of Internal Medicine, University of Oklahoma Health Sciences Center, Oklahoma City, OK 73104, USA

4 Department of Biochemistry and Molecular Biology, University of Oklahoma Health Sciences Center, Oklahoma City, OK 73104, USA 
exposing the underlying lamina propria to luminal microbiota. Concomitantly, chronic epithelial repair responses are induced to replace damaged/dying epithelium [1]. However, the role of epithelial repair in the pathogenesis of UC is unclear. Further, the cell types contributing to this response are also elusive.

Colonic epithelium is renewed every $4-5$ days in humans (1-4 days in mice) [5]. Physiological maintenance of the colon epithelium is derived from colonic epithelial stem cells $[6,7]$. Currently, the best-characterized stem cell population is that marked by leucine-rich G-protein coupled receptor $(\mathrm{Lgr}) 5[8,9]$. $\mathrm{Lgr}^{+}$cells can give rise to all known epithelial lineages in the small and large intestine in vivo [10], and in vitro [11, 12]. However, it is now known the $\mathrm{Lgr}^{+}$population is not indispensible owing to considerable plasticity among epithelial stem cell types and existence of other populations capable of producing progeny [13-15]. Further, it is unclear which among these functional stemlike cells contributes to epithelial recovery following acute and chronic intestinal insult.

Recent work has highlighted a unique population of epithelial cells, called tuft cells, in regulating epithelial responses to injury [16]. Tuft cells belong to an epithelial lineage distinct from goblet, enteroendocrine, absorptive, and Paneth cells [17]. They are characterized by their unique morphology and strong specific expression of double cortin-like kinase 1 (Dclk1) [18]. These cells have emerged as important sentinels in the intestinal tract that guide host responses to specific injury, including host defense against helminth infection [19-23], promoting epithelial repair after acute intestinal injury and tumorigenesis [16].

Despite these new functions ascribed to tuft cells, mechanisms underlying their abilities are still unclear. Recent studies have shown that Dclk1 itself may mediate some of these functions. In the context of acute intestinal injury caused by dextran-sodium sulfate (DSS) or radiationinduced acute small intestinal injury, epithelial ablation of Dclk1 worsened disease outcome, due to failure to promote protective intestinal epithelial regeneration [24, 25]. However, these models are limited in that they rely on acute exposure to cytotoxic agents (DSS or ionizing radiation) that cause sudden catastrophic insult to epithelium. Such a scenario provides limited insights into a clinical situation characterized by spontaneous induction and maintenance of inflammation as observed in IBD. Thus, the role of Dclk1 in spontaneous and chronic microbiota-dependent inflammatory diseases such as UC is unknown.

We have developed a model of spontaneous, chronic colitis that is induced by destabilization of the colonic mucus barrier in the presence of the microbiota [26]. This mucus layer defect is due to gene-targeted knockdown of the key glycosyltransferases C1GalT1 and C3GnT that modify the Muc2 mucin with $O$-linked oligosaccharides which protect the Muc2 protein core from luminal proteases, allowing it to sequester the bacteria in the lumen and limit their interaction with the distal colon mucosa $[26,27]$. These $O$-glycan deficient mice develop microbiotadependent distal colitis within the first 2 weeks of life that remains and increases in severity during the life of the mouse [26]. The disease recapitulates features observed in human UC, including disease initiation and severity localized to the rectum, massive influxes of immune cells, crypt abscesses, surface epithelial damage, and constitutive inflammation-induced epithelial repair [28]. These mice therefore make an ideal model to study the role of Dclk1 in the setting of abnormal host-bacterial interactions that lead to chronic UC-like disease.

We tested the hypothesis that tuft cells, via Dclk1, facilitate the renewal of intestinal epithelium during chronic inflammation, using our spontaneous colitis model. Our results demonstrate that epithelial-specific ablation of Dclk1 impairs overall epithelial repair responses elicited by bacteria-induced inflammation, supporting an important role for Dclk1-positive tuft cells in promoting epithelial restitution during chronic injury.

\section{Materials and methods}

\section{Mice}

Mice lacking intestinal mucin-type core 1 O-glycans $\left(\right.$ Clgalt ${ }^{f f f}$; VillinCre $)$ were bred with mice lacking core 3 O-glycans $\left(C 3 G n T^{-1-}\right)[26,27]$ to generate mice deficient in both O-glycan types (DKO) [26]. DKO mice were crossed with $D c l k l^{f f f}$ mice to generate mice additionally lacking intestinal epithelial Dclk1 (DKO;Dclk1 $1^{\Delta I E C}$ ). Mice deficient only in Dclk1 in epithelium (IEC Dclk1 ${ }^{-/-}$or $D c l k 1^{\Delta I E C}$ ) were generated by breeding Dclkl fff with VillinCre Tg mice. All mice are on a C57BL/6J and $129 \mathrm{~Sv}$ mixed background. Littermates were used as controls. Healthy controls were defined as VillinCre-negative Clgaltff $; C 3 G n T^{+/-}$or ${ }^{f-} ; D c l k l^{f / f}$ or f/w. . Mice were housed in a specific pathogen-free facility and fed standard chow (PicoLab Rodent Diet 20). All animal procedures were approved by the institutional animal care and use committee (IACUC).

\section{Tissue preservation and histology}

For histology, colon tissues were harvested immediately after euthanization and fixed with $10 \%$ neutral buffered formalin or in ice-cold Carnoy's fixative $(60 \%$ methanol, $30 \%$ chloroform, and $10 \%$ acetic acid) prior to paraffin embedding. Paraffin-embedded sections $(5 \mu \mathrm{m})$ were 
stained with hematoxylin and eosin, or periodic acid-Schiff (PAS) to stain glycogen and mucin [26]. Pictures were taken with a Nikon Eclipse E600 microscope equipped with a Nikon DS-2Mv camera, using the software NIS Freeware 2.10 (Nikon Instruments; Lewisville, TX). Histology was scored as previously described [26].

\section{Immunostaining}

For cryopreservation, the colon tissues were fixed in $4 \%$ paraformaldehyde at $4{ }^{\circ} \mathrm{C}$ overnight, rinsed in PBS, placed in $20 \%$ sucrose in PBS for $2 \mathrm{~h}$, and then embedded in a 50:50 mixture of optimal cutting temperature (OCT) compound (Sakura Finetek; Torrance, CA) and tissue freezing medium (Electron Microscopy Sciences; Hatfield, PA), and finally frozen in dryice- cooled isopentane. Cryosections (10 $\mu \mathrm{m}$ thick) were used for immunostaining. Formalin or Carnoy's-fixed paraffin-embedded sections were deparaffinized and rehydrated. As required (e.g., for Ki67, Dclk1, Muc2 staining), antigen retrieval was performed by heating sections between 95 and $100{ }^{\circ} \mathrm{C}$ for $23 \mathrm{~min}$ in Antigen Unmasking Solution (Vector Labs). Blocking of nonspecific antibody binding was performed using serum-free protein block (DAKO; Carpinteria, CA; $10 \mathrm{~min}$, RT). Following blocking, sections were incubated with primary antibody in a humidified chamber overnight at $4{ }^{\circ} \mathrm{C}$, or $2 \mathrm{~h}$ at ambient temperature. The primary antibodies used were rabbit antiDclk1 (ab31704, 1:2000); goat anti-Cox1 (Santa Cruz, sc1754, 1:100); rabbit anti-Ki67 (ThermoScientific RM-9106S0, 1:100); rat anti-F4/80 (clone Cl:A3-1; Bio-Rad MCA497G, 1:200); rabbit anti-myeloperoxidase (Thermo Fisher Scientific, RB-373-A0, 1:200); mouse anti-PCNA (Abcam, ab29, 1:2000); rabbit anti-MUC2 (Abcam ab9007, 1:200); or rabbit anti-E-cadherin (Santa Cruz, sc-7870, 1:200). Following washing with PBS-Tween $(0.05 \%$ Tween-20 in $1 \times$ PBS), sections were incubated with the appropriate secondary antibodies (AF594-labeled donkey anti-rabbit IgG, AF488- or AF555-labeled donkey anti-goat IgG, AF488-labeled goat anti-rabbit IgG, AF488-labeled donkey anti-rat $\mathrm{IgG}$ ) at a concentration of $8 \mu \mathrm{g} / \mathrm{mL}$ for $45 \mathrm{~min}$ at RT, and then counterstained with $25 \mathrm{ng} / \mathrm{mL} \mathrm{4,6-}$ diamidino-2-phenylindole (DAPI) in PBS for $5 \mathrm{~min}$, washed, and mounted using PermaFluor antifade (ThermoScientific). Isotype control antibodies or nonprimary antibody-treated sections were used for negative controls. For epifluorescent imaging, specimens were analyzed with a Nikon Eclipse 80i microscope equipped with a Nikon DSQi1MC camera operating through NIS Elements AR software (version 3.0). For confocal imaging, a Nikon Confocal C2 microscope using NIS Elements was used, and z-stacks were obtained from slices $0.3 \mu \mathrm{m}$ apart through the thickness of the section. Confocal images were processed using Imaris software (V7.7.2).

\section{Fluorescence in situ hybridization (FISH) for bacteria and co-staining with Muc2}

FISH and dual staining for FISH and Muc2 was carried out as described [26]. For bacterial penetration depth analysis, the distance of EUB $338^{+}$cells from the mucosal surface was measured using Image $\mathbf{J}[29]$, with the line perpendicular to the surface. The surface mucosa is given an arbitrary distance of zero; measurements above the plane are given a positive value; those below the plane were given a negative value.

\section{Crypt isolation and culture of colonic organoids}

Crypts were isolated as described [28]. Isolated crypts were pelleted, washed with cold HBSS buffer, and centrifuged at $50 \mathrm{~g}$ for $5 \mathrm{~min}$ to separate crypts from single cells, and counted using a hemocytometer. Crypts were embedded in Matrigel on ice (growth factor reduced, with phenol red; Corning 354234) and seeded in a 24-well plate (600 crypts/ $15 \mu \mathrm{L}$ of Matrigel/well). The matrigel was polymerized for $2-5 \mathrm{~min}$ at $37^{\circ} \mathrm{C}$. For standard culture, $500 \mu \mathrm{L} / \mathrm{well}$ of basal culture medium (Advanced Dulbecco's Modified Eagle medium/F12 supplemented with penicillin/streptomycin, $10 \mathrm{mM}$ HEPES, glutamine, $1 \times \mathrm{N} 2,1 \times \mathrm{B} 27$, and $1 \mathrm{mM} \mathrm{N}$ acetylcysteine, $10 \mu \mathrm{M}$ Y27632 ROCK kinase inhibitor, $10 \mathrm{mM}$ nicotinamide, $10 \mu \mathrm{M}$ SB431542, murine Wnt3a $(0.1 \mu \mathrm{g} / \mathrm{mL})$, mouse EGF $(0.05 \mu \mathrm{g} / \mathrm{mL})$, mouse Noggin $(0.1 \mu \mathrm{g} / \mathrm{mL})$ and human R-spondin $(0.5 \mu \mathrm{g} / \mathrm{mL})$ was added, and organoids were cultured at $37^{\circ} \mathrm{C}$. For conditioned media, we followed Miyoshi et al. [30]: Briefly, the Wnt3a, Noggin, and R-spondin-secreting murine fibroblast line L-WRN (ATCC-CRL3276) [30] was propagated in DMEM containing 10\% EquaFETAL (Atlas Biologicals). After 90$100 \%$ confluence, cells were cultured in primary culture medium (PCM; Advanced DMEM: F12 media with 20\% FBS (Atlas Biologicals), and 1× Glutamax (Gibco), 1× penicillin/streptomycin (Gibco)) for $24 \mathrm{~h}$ to generate the conditioned primary culture media (CPCM), which was mixed 1:1 with PCM (generating 50\% CPCM) and stored at $-20{ }^{\circ} \mathrm{C}$ until use. Subsequently, crypts were isolated from distal colons as above, and cultured with 50\% CPCM in matrigel. For the initial culture, $10 \mu \mathrm{M}$ Y 27632 was added to $50 \%$ CPCM to reduce primary epithelial cell death [30], but was not used for later maintenance, passage, or treatment of organoids. At various timepoints, images were taken using an EVOS Cell Imaging System (Thermo Fisher Scientific).

\section{Organoid treatment}

For prostaglandin E2 (PGE2) treatment, organoids were treated with $1 \mu \mathrm{M}$ of either PGE2 or 16,16-dimethyl PGE2 (dmPGE2) (both from Cayman Chemicals) for $48 \mathrm{~h}$, and imaged and harvested for proliferation analysis as described 
below. For inhibition of Wnt signaling pathways, two strategies were employed: First, to remove the Wnt signal while keeping the pathway intact within organoids, we treated cultured spheroids or colonoids with Wnt-depleted $50 \%$ CPCM. This was generated similar to Miyoshi et al. [31], by setting aside a flask of L-WRN cells and culturing them in PCM containing $20 \mu \mathrm{M}$ C59 Wnt inhibitor (Cayman Chemicals) overnight and mixing this 1:1 with PCM as above. Conditioned media from the same batch of cells was used as a control. $24-48 \mathrm{~h}$ after treatment, organoids were imaged and harvested for downstream processing. Second, a complementary approach was used to specifically inhibit the Wnt pathway in organoids in the presence of Wnts, whereby established spheroids or colonoids were treated with $10 \mathrm{ng} / \mathrm{mL}$ IWR1-endo (Cayman Chemicals) in $50 \%$ CPCM, cultured for $24-48 \mathrm{~h}$, and imaged and harvested for embedding as described.

\section{Organoid processing}

Organoids were processed similar to Fernando et al. [32]. Organoid-containing matrigel was collected by mechanical disruption and aspiration from the well with a pipette together with wash media (DMEM/F12 with $10 \%$ FBS, and $1 \times$ penicillin/streptomycin), transferred to a $1.5 \mathrm{~mL}$ microtube, and centrifuged $(300 \times g)$. The supernatant was discarded and the pellet was resuspended in 4\% PFA and fixed for $10 \mathrm{~min}$. 4\% Ultrapure $^{\mathrm{TM}}$ low melting point (LMP) agarose (Invitrogen) was then mixed with fixed matrigel/organoids $1: 1(\mathrm{v} / \mathrm{v})$, and transferred into the middle of a $\sim 40{ }^{\circ} \mathrm{C}$ presolified $4 \%$ LMP agarose layer in a cryomold, and then overlayed with fresh LMP agarose to form a "sandwich" structure. The agarose block was fixed with $4 \%$ PFA overnight at $4{ }^{\circ} \mathrm{C}$, and then processed for paraffin-embedding. $5 \mu \mathrm{m}$-thick sections were collected for histology and immunostaining as described above.

\section{PGE2 production}

PGE2 production of colon organ culture supernatants and colon tissue lysates was analyzed by ELISA (PGE2 ELISA Kit, Endo Life Sciences). Colon tissue was cultured as previously described [28]. Briefly by $\sim 3 \mathrm{~mm}$ pieces of distal colon tissues were cultured in RPMI media (1-2 mL) containing antibiotics at $37^{\circ} \mathrm{C}$ for $24 \mathrm{~h}$. The supernatant was cleared by centrifugation, and supplemented with $1 \times$ Halt $^{\oplus}$ Protease Inhibitor (Thermo Fisher Scientific) for analysis. The remaining colonic tissue was weighed for normalization. Colon tissue lysate was prepared as previously described [28] by lysing tissues in RIPA buffer with a Minibead beater (Zirconia/silica beads), and quantified using the Dc Protein Assay (Biorad). For tissue lysates, protein was normalized to $0.0013 \mathrm{mg} / \mathrm{mL}$ among all samples, and 0.13 $\mu \mathrm{g}$ was used for ELISA.

\section{RNA extraction and quantitative reverse- transcription polymerase chain reaction}

Total RNA was extracted from colon tissues and isolated epithelium using the RNeasy Plus Kit (Qiagen). $1.5 \mu \mathrm{g}$ of RNA was used for complementary (c)DNA synthesis using OmniScript Reverse Transcriptase kit (Qiagen) for subsequent quantitative polymerase chain reaction (qPCR). For all qPCR, SsO Advanced Reaction Mix (Bio-Rad) was used and qPCR was performed using a BioRad CFX96 RealTime instrument. Reaction conditions for genes tnfa, ill7a, nos2, ccndl, myc, are previously described [28]. Primer sequences and reaction conditions for ifng, il22, notchl, hes1, atohl, klf5, lgr5, axin, ptgs2 are described in Supplementary Table 1.

\section{Statistics}

All data are presented as mean $\pm \mathrm{SD}$, unless otherwise indicated. Paired Student's $t$-test (two-tailed) was used to analyze the mean differences between littermate pairs. Oneway analysis of variance (ANOVA) followed by Bonferroni post-test was used to analyze the difference between 3 or more groups. The D'Agostino and Pearson omnibus K2 tests were used to determine whether data fit a normal distribution. A $P$ value $<0.05$ was considered to be significant. "\#” indicates Paired $t$-test; “*” or "†" indicate ANOVA. Data were plotted and analyzed using GraphPad Prism version 7.0. Power analysis was determined by pilot studies with similar mouse numbers. Unless otherwise indicated in figure legends, all experiments used $n=5$ mice per group, and are representative of 2 independent experiments. For in vivo studies, reported sample number per experiment represent biologic replicates (comparison of animals of one genotype with littermates of another genotype). Both male and female mice were used in the study. For primary cell culture assays, samples were assayed in duplicate, and technical replicates were pooled prior to analysis. Sample data collection represents mean of 10-30 organoids from each genotype per experiment. Where possible, organoids from littermates were compared.

\section{Results}

\section{Deletion of Dclk1 specifically in intestinal epithelium exacerbates spontaneous bacterial-dependent colitis}

To test the role of Dclk1 in intestinal epithelial repair during chronic inflammation, we utilized our recently characterized DKO model, which develop spontaneous chronic bacterialdependent colitis, resembling human UC $[26,27,33]$. We crossed these DKO mice with $D c l k{ }^{f f f}$ mice to generate 
DKO;Dclk $1^{\Delta I E C}$ mice (Fig. 1a). DKO;Dclk1 ${ }^{\Delta I E C}$ mice were viable, and we initially let them age to 1 month, when colitis is established in DKO mice [26], and analyzed WT control, $D c l k 1^{\Delta I E C}$, DKO, and DKO;Dclk1 ${ }^{\Delta I E C}$ mice for signs of disease. As expected, DKO mice demonstrated heightened indexes of clinical disease vs. control mice, including softer stool and reduced body weight; however, these parameters were worsened in DKO;Dclk1 $1^{\Delta I E C}$ littermates (Fig. 1b). Histology of the distal colon (last 3rd of colon) revealed clinical disease was accompanied by robust colitis in DKO
A

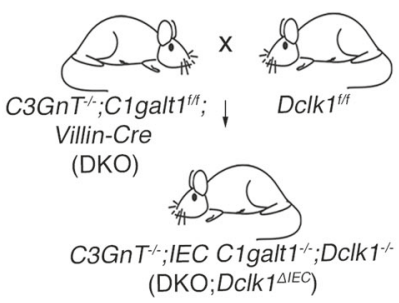


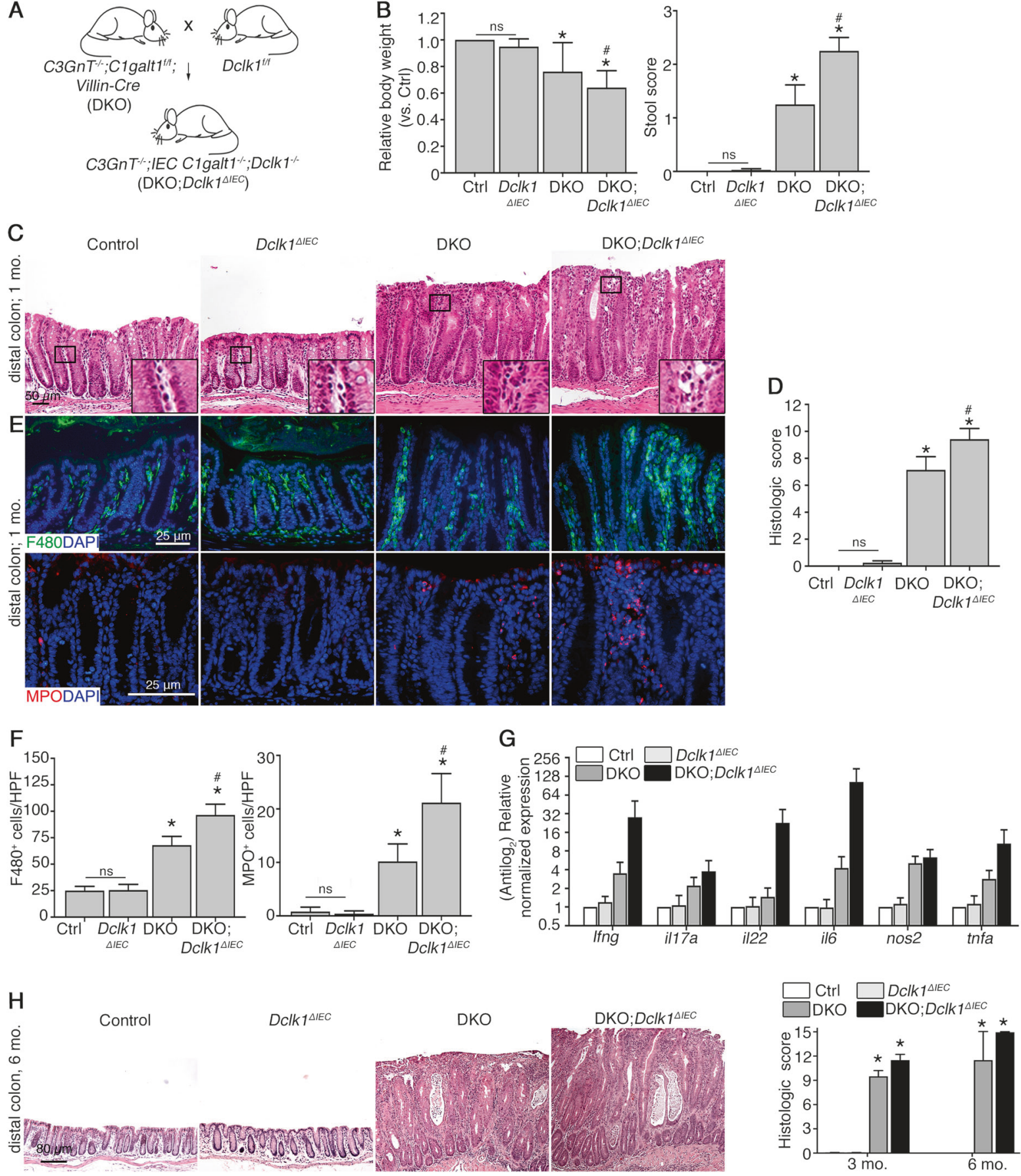

Fig. 1 Conditional deletion of Dclk1 in gut epithelium worsens spontaneous bacterial-dependent colitis. a Schematic diagram showing derivation of $D K O ; D c l k 1^{\Delta I E C}$ mice. b Quantification of relative body weight and stool score. c H\&E staining of murine distal colons at indicated timepoints. Inset: magnification of boxed regions. d Histologic scores of disease. e Epifluorescent staining on FFPE tissue sections. f Quantitation of inflammatory cell populations in rectal tissues. g qPCR for inflammatory gene expression in distal colonic tissue. h H\&E staining of murine distal colons. Graph: histologic colitis scores. For $(\mathbf{a}-\mathbf{d})$, results are representative of 7-8 mice/group $(\sim 1$ month-old). For (e-h), results are representative of 4 mice/group. $* P<$ 0.05 vs. Ctrl and $D c l k 1^{\Delta I E C}$; ${ }^{\#} P<0.05$ vs. DKO. ns non-significant 
mice but not controls; however, colitis was exacerbated in DKO;Dclkl ${ }^{\Delta I E C}$ mice, which showed enhanced mucosal thickening, and increased inflammatory cell infiltration vs. DKO littermates (Fig. 1c, d). Mice singly deficient in gut epithelial Dclk1 (Dclkl ${ }^{\Delta I E C}$ mice) did not exhibit any overt signs of disease, consistent with previous studies (Fig. 1bd) [25].

The worsened disease in $\mathrm{DKO} ; \mathrm{Dclk1} \mathrm{IIEC}^{\Delta e l a t i v e}$ to DKO mice suggested an altered inflammatory response during chronic colitis in the absence of Dclk1. Further immunostaining using macrophage marker F4/80, and neutrophil marker MPO revealed that both cell types are most abundant during inflammation, but most heavily infiltrate the lamina propria of $\mathrm{DKO} ; \mathrm{Dclk1} 1^{\Delta I E C}$ mice vs. the other mouse lines, with macrophages predominating (Fig. 1e, f). qPCR analysis showed a comparable induction of ifng, thfa, ill7a, and nos 2 in DKO;Dclk1 $1^{\Delta I E C}$ and DKO littermates vs. control mice, although a trend toward increased proliferation-inducing cytokines il6 and il22 within inflamed tissues lacking Dclk1 (Fig. 1g). Loss of Dclk1 alone had no bearing on number of immune cells or inflammatory gene expression at baseline (Fig. 1e-g). These studies suggest that Dclk1 expression influences the magnitude but not the type of immune response in chronic colitis. Analysis of older mice ( 3-6 months) revealed DKO;Dclkl $1^{\triangle I E C}$ mice consistently maintained a worsened colitis state vs. DKO littermates (Fig. 1h). Collectively, these studies reveal a protective function of Dclk1 in the colonic mucosa in response to spontaneous chronic colitis.

\section{Dclk1 affects goblet cell differentiation status during inflammation}

We noted the increased mucosal damage in inflamed tissues lacking Dclk1 was associated with altered crypt architecture at high magnification (Figure S1A). We hypothesized this reflected a change in the differentiation status of the epithelium in the absence of Dclk1 during inflammation. We tested this by examining the goblet cell lineage via histochemical staining with PAS to detect mucin-rich mature goblet cells [34], and immunostaining for goblet cellspecific Muc2. PAS staining showed DKO mice had abundant smaller goblet cells vs. control mice as expected; however, DKO;Dclk1 ${ }^{\Delta I E C}$ mice displayed fewer goblet cells than DKO, being similar to controls, and in some regions further exhibited larger amounts of secreted mucin within crypt lumens in DKO;Dclk1 $1^{\Delta I E C}$ vs. DKO and healthy control mice (Figure S1B). Muc2 staining and quantification of $\mathrm{Muc}^{+}$cells confirmed the goblet cell phenotype (Figure S1C and D). In contrast, we found goblet cells in $D c l k 1^{\Delta I E C}$ mice did not exhibit any obvious defect without inflammation (Figure S1A-D). These results suggest loss of Dclk1 affects differentiation and function of non-tuft cell populations only during inflammation. To gain insight into the differentiation pathways which might be affected, we obtained mRNA from distal colon crypts for expression analysis of key genes governing goblet cell differentiation, including notchl [35], hes 1 [36], atohl [37], and klf4 [38, 39]. We found that regardless of Dclk1 status, inflamed tissues exhibited heightened Notch1 expression, which is a negative regulator of goblet cell differentiation (8); however, Dclk1 expression during inflammation was associated with reduced Notch1 target hes1, as well as klf4, the latter which directs goblet cell maturation (Figure S1E) [38]. Given spontaneous inflammation in the absence of a mucus layer is bacterial-dependent [26, 27], we compared hostmicrobe interactions in situ by dual staining for Muc2 and the universal bacterial $16 S$ rRNA gene probe EUB338 to determine if the goblet cell phenotype impacted the mucus barrier. Control and $D c l k 1^{\Delta I E C}$ mice showed a functional mucus barrier separating luminal microbiota from the epithelium, whereas DKO and DKO;Dclk1 $1^{\Delta I E C}$ mice exhibited a defective mucus layer with bacteria directly contacting the epithelium (Figure S1F and G). Collectively, these studies indicate a role for Dclk1 in regulating goblet cell differentiation in vivo during inflammation. However, the modest overall impact of Dclk1 on this aspect of epithelial function led us to reason this kinase was involved in regulating mucosal responses through alternative mechanisms.

\section{Absence of Dclk1 signaling impairs epithelial regenerative responses induced by microbiota- dependent chronic inflammation}

It is known Dclk1 expression is important to induce regenerative responses in models of acute intestinal injury [25]. However, since inflammatory cytokines can also promote regeneration [40, 41], it is unclear how this aspect of epithelial function was affected in the absence of Dclk1. To address this, we performed Ki67 staining on colonic sections, finding that the crypt proliferative $\left(\mathrm{Ki}_{6}{ }^{+}\right)$compartment in DKO;Dclk1 ${ }^{\Delta I E C}$ mice was significantly reduced vs. DKO littermates, comparable to control and $D c l k l^{\Delta I E C}$ mice (Fig. 2a, b). Dclk1 function has been linked to the expression of genes regulating proliferation [42], and our previous studies reveal that epithelial hyperproliferation is associated with increased activation of $\mathrm{Wnt} / \beta$-catenin pathways in DKO mice [28], suggesting the defect in DKO; $D c l k 1^{\Delta I E C}$ mice may be linked to Wnt signaling. Consistent with this, qPCR of mRNA extracted from crypt epithelium of control, Dclk1 $1^{\Delta I E C}$, DKO, and DKO;Dclk1 $1^{\Delta I E C}$ mice showed a significant reduction in Wnt-dependent pro-proliferative gene $c c d n 1$ (cyclin D1), and a trend for reduced axin2, $\lg 5$, and $m y c$ expression, in inflamed tissues of DKO;Dclk1 $1^{\Delta I E C}$ vs. DKO littermates, to levels near control and $D c l k 1^{\Delta I E C}$ mice (Fig. 2c). These data suggest a 
A

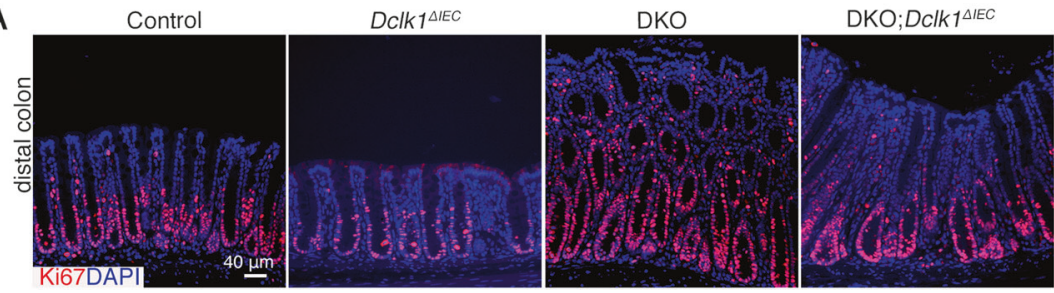

B
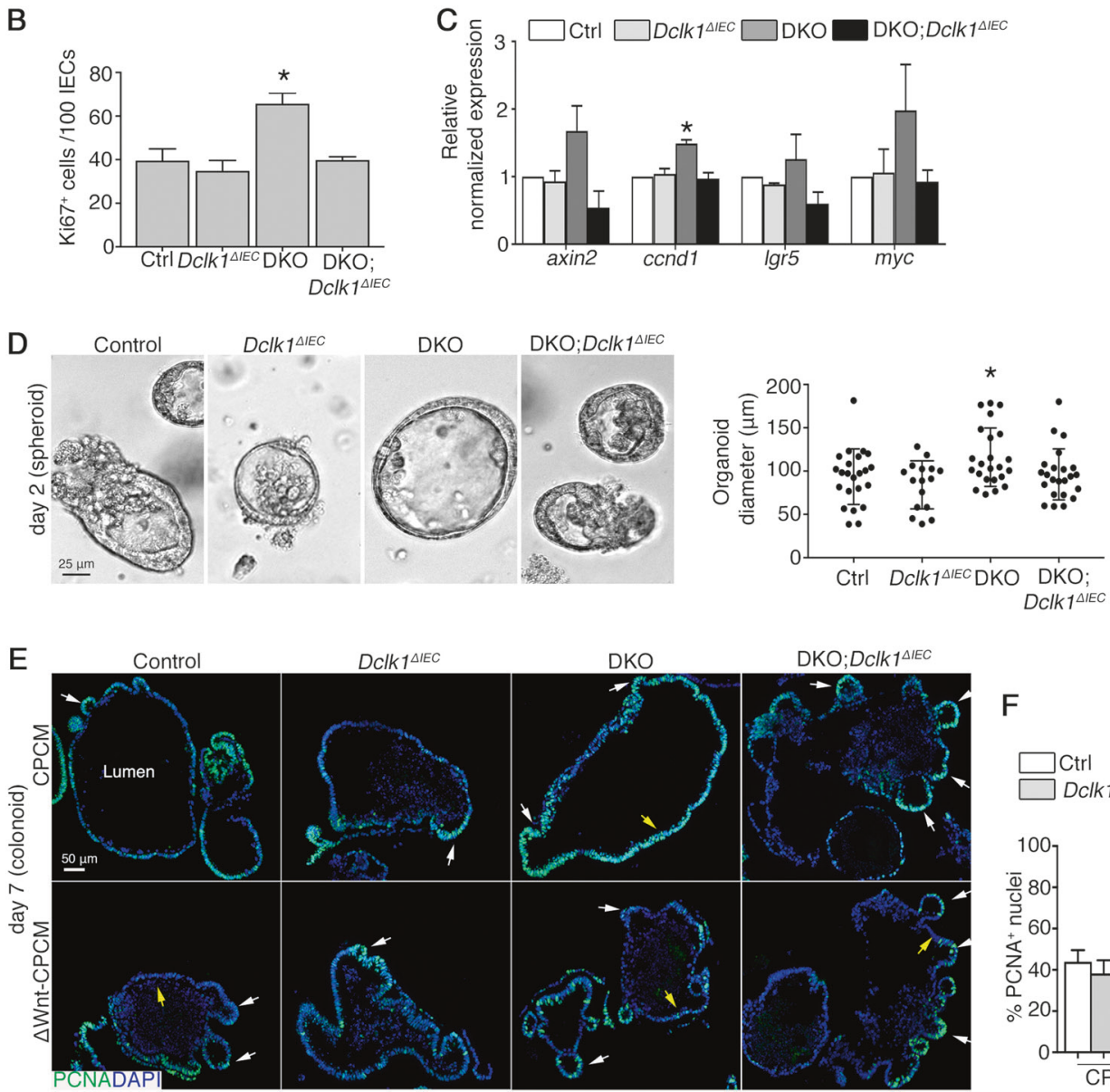

$\mathrm{F}$
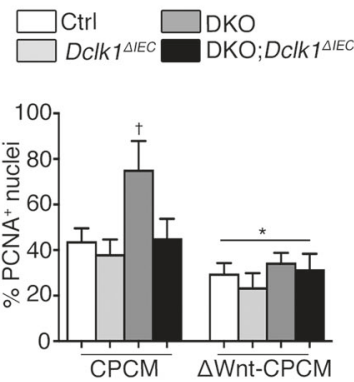

Fig. 2 Dclk1 contributes to microbiota-dependent chronic inflammation-induced epithelial repair responses in vivo and ex vivo. a IF staining on colon tissue. b Mean number of Ki67 ${ }^{+}$cells in distal colon crypts. $\mathbf{c}$ qPCR for Wnt-regulated genes in colonic crypts. Error bars, S.E.M. d Micrograph of a representative spheroid in a 3D matrigel system. Graph on right: spheroid diameter quantification; each point represents a single spheroid. e IF staining on FFPE

significant role for Dclk1 in regulating epithelial proliferative responses to chronic inflammatory stimuli.

It was unclear whether the effect of epithelial Dclk1 on proliferation was due to an intrinsic defect within epithelium or secondary to the inflammatory microenvironment. To address this, we harvested crypts from 1-month-old WT, $D c l k 1^{\Delta I E C}$, DKO, and DKO;Dclk1 ${ }^{\Delta I E C}$ mice for spheroid culture [30], and compared spheroid size, growth rates, proliferation, and differentiation status over 4 days. At days 2 and 4 of culture, we found DKO spheroids were largest in diameter, while control, Dclk1 ${ }^{\Delta I E C}$, and DKO;Dclk1 $1^{\Delta I E C}$ colonoids. White arrows, colonoid buds; Yellow arrows, cells lining colonoid lumen. $\mathbf{f}$ Enumeration of $\mathrm{PCNA}^{+}$nuclei of colon organoids. For $(\mathbf{a}-\mathbf{c})$, results are representative of 7 mice/group, $\sim 1$ month of age. For $(\mathbf{e}, \mathbf{f})$, results are representative of 3 independent experiments, with organoids from each group cultured in duplicate. For $(\mathbf{b}-\mathbf{d}), * P<0.05$ vs. all other groups. For (f): ${ }^{\dagger} P<0.05$ vs. all other vehicle-treated groups; $* P<0.05$ vs. corresponding genotype in CPCM group

mice all showed similar diameter (Fig. 2d and Figure S2A). The increased size and budding in DKO spheroids was associated with increased proportion of proliferating $\left(\mathrm{PCNA}^{+}\right)$cells vs. control, Dclk1 $1^{\Delta I E C}$ and DKO;Dclk $1^{\Delta I E C}$ spheroids at both timepoints, whereas proliferation status of DKO;Dclk $\left.\right|^{\Delta I E C}$ spheroids was comparable to control and $D c l k 1^{\Delta I E C}$ mice (Figure S2B). H\&E stained cross-sections of spheroids derived from all mouse lines revealed intact spheroid structure with visible lumen and polarized epithelium, with the diameter greatest in DKO spheroids consistent with live cell imaging (Figure S2C). Similar 
results were shown in crypts cultured in conditioned media [30], or derived from older mice (3-5 months) (not shown). These studies suggest chronic inflammation interacts with Dclk $1^{+}$cells to prime epithelium for sustained proliferative responses even in the absence of inflammatory stimuli.

Since spheroids are cultured in stem cell-enriched conditions due to the addition of their maintenance factors, we further explored how Dclk1 function related to stem cell activity. We addressed this by inhibiting the Wnt pathway, which is required for the maintenance of colonic stem cells [12], in control, Dclk1 $1^{\Delta I E C}$, DKO, and DKO;Dclk1 $1^{\Delta I E C}$ spheroids that have matured to colon organoid (colonoid) cultures, in which crypt-like buds are prolific (5 days culture) (Figure S3A-C). Here the stem cells locate to the base of the crypt bud, and give rise to immature progenitors and differentiated cells lining the colonoid lumen [12, 43, 44]. Notably, the asymmetric colonoid morphology abolished overall size differences seen in the spheroid state above between DKO, and DKO;Dclk1 ${ }^{\Delta I E C}$-derived cultures (Figure S3B). To observe the relationship of stem cell compartmentalization with overall proliferative capacity of colonoids, we treated colonoids at day 5 of culture for 2 days with Wnt3a-rich (50\% CPCM) or Wnt3a-depleted media (50\% CPCM lacking Wnt proteins; see Materials and Methods), and harvested organoids for sectioning and staining with PCNA (Figure S3A). We noted Wnt inhibition did not affect colonoid budding number (Figure S3B and C). However, we found in Wnt3arich conditions, DKO organoids remained in a highly proliferative state in both stem and non-stem compartments (Fig. 2e). The total number of proliferating cells was significantly increased vs. littermate DKO;Dclk $1^{\Delta I E C}$ colonoids, which were similar to control and Dclk $1^{\Delta I E C}$ mice in total number of $\mathrm{PCNA}^{+}$cells, and of localization of the proliferative compartment to crypt buds (Fig. 2e (upper panel), f). In contrast, Wnt depletion significantly reduced proliferation in all groups, most dramatically in DKO colonoids, ultimately normalizing rates and confining the remaining dividing cells to the bud regions in all groups (Fig. 2e (lower panel), f). Similar results were shown in colonoids treated with the Wnt signaling inhibitor IWR1-endo in Wnt-rich CPCM, confirming the specificity of the Wnt pathway in this system (Figure S3D). Wnt inhibition also caused a significant drop in stem cell-enriched spheroid diameters (Figure S3E-G). These results suggest inflammation primes both stem and especially stem-cell-derived progenitor cells for Wnt sensitivity in a Dclk1-dependent manner.

\section{The reduced inflammation-associated proliferative response caused by loss of Dclk1 is linked to defective PGE2 production in the colonic mucosa}

Previous studies of the small intestinal epithelium demonstrated Dclk1 signaling by tuft cells is a critical modulator of stem cell activity and tissue repair after total body irradiation [45]. One primary defect was a significant reduction of Cox 2 expression in the absence of epithelial Dclk1, leading to reduced synthesis of the lipid metabolite prostaglandin-E2 (PGE2) [45], which is a known modulator of epithelial proliferation in the colon [46-48]. To determine if Dclk1 also contributed to this pathway during colitis, we analyzed Ptgs 2 (Cox2) mRNA expression by qPCR and total PGE2 protein levels by ELISA. Ptgs 2 expression in the DKO;Dclk1 ${ }^{\Delta I E C}$ colon was significantly reduced compared to the DKO colon, to levels near control and $D c l k 1^{\Delta I E C}$ mice (Fig. 3a). Consistent with the Ptgs2 expression data, PGE2 levels in DKO;Dclk1 ${ }^{\Delta I E C}$ colons were significantly reduced compared with DKO colons, to levels near control and $D c l k l^{\Delta I E C}$ mice (Fig. 3b). To determine if PGE2 could rescue proliferation defects in DKO;Dclkl $1^{\Delta I E C}$ epithelium, we treated spheroids (day 2 of culture) of different genotypes with PGE2 and compared their responses to vehicle (DMSO)-treated spheroids. After 2 days, PGE2 significantly induced growth of DKO; $D c l k 1^{\Delta I E C}$ spheroids relative to vehicle-treated spheroids (Fig. 3c). Similar results were observed in response to treatment with 16,16-dimethyl PGE2 (dmPGE2), a stable derivative of PGE2 [31] (Figure S4A and B). To determine the relationship of this growth phenotype to proliferation and stem-cell compartmentalization, we let spheroids mature to colonoids as above (day 5 primary culture), treated them for 2 days with vehicle or PGE2, and harvested them for proliferation analysis. Our results show that PGE2 treatment significantly increased the number of $\mathrm{PCNA}^{+}$ cells of DKO;Dclk1 ${ }^{\Delta I E C}$ colonoids vs. vehicle-treated colonoids, with proliferating zones expanding beyond the buds into the luminal epithelium (Fig. 3d). To determine the relationship of PGE2-stimulated proliferation to the Wnt $\beta$ catenin pathway, we stained colonoids with active $\beta$-catenin, finding increased active $\beta$-catenin staining within cells in PGE2-treated vs. vehicle-treated DKO;Dclk $1^{\Delta I E C}$ organoids (Figure S4C). Further, Wnt-depletion abolished the ability of PGE2 to increase spheroid growth (Figure S4DF). Collectively, these studies support that Dclk1 signaling during chronic inflammation contributes to inflammationinduced proliferation via Cox2-dependent PGE2 production, leading to enhanced Wnt signaling activity downstream in colonic stem and progenitor cells.

\section{Dclk1 influences tuft cell activation but not expansion during chronic colitis}

In the colon mucosa, Dclk1 is mainly found in tuft cells, which also express Cox $1[18,24]$. To determine the relationship of disease severity with Dclk1 expression and the tuft cell population, we examined the expression pattern of Dclk1 and Cox 1 on formalin-fixed paraffin-embedded colon 

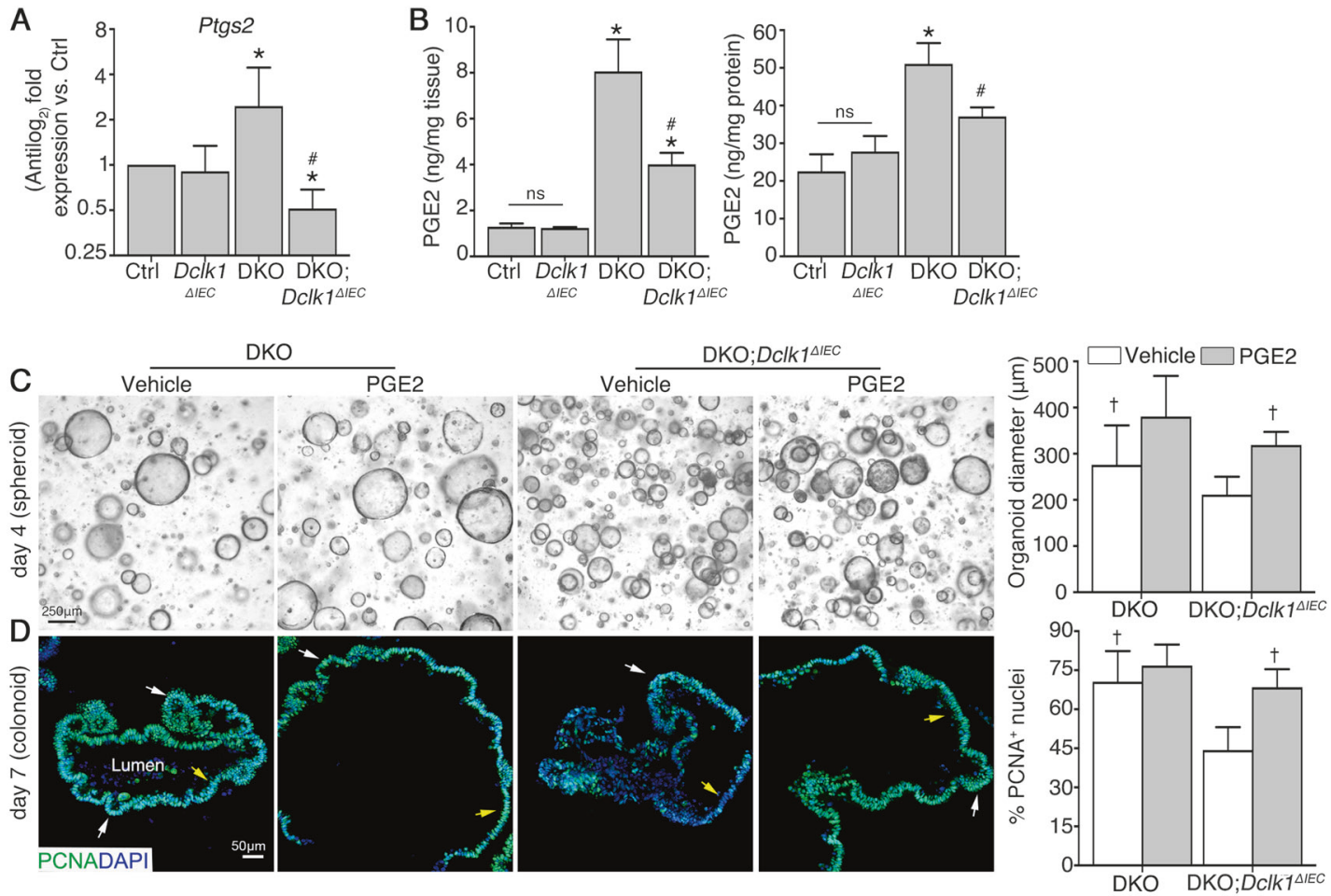

Fig. 3 The reduced colitis-associated proliferative response caused by loss of Dclk1 is linked to defective PGE2 production in colonic mucosa. a qPCR for the gene encoding Cox 2 in distal colonic tissues. b Quantitation of PGE2 production from distal colon organ culture supernatants (right) or tissue lysates (left). c Micrograph of representative spheroids in a 3D matrigel system. Graph on right: spheroid diameter quantification. d IF staining on FFPE colonoids. Graph on right: Enumeration of $\mathrm{PCNA}^{+}$nuclei of colonoids. For $(\mathbf{a}, \mathbf{b})$, results are representative of 5 mice/group. For $(\mathbf{c}, \mathbf{d})$, results are representative of 3 independent experiments, with organoids from each group cultured in duplicate. ${ }^{*} P<0.05$ vs. control and $D c l k 1^{\Delta I E C}$; ${ }^{\#} P<0.05$ vs. DKO. ${ }^{\dagger} P<0.05$ vs. vehicle-treated DKO;Dclk1 $1^{\Delta I E C}$ tissue sections in 1-month-old control, DKO, and DKO; $D c l k 1^{\Delta I E C}$ mice. As expected, dual staining of Dclk1 and Cox1 labeled a discrete cell population in crypt epithelium of control and DKO mice, with a distinct morphology consistent with the tuft cell population [18] (Fig. 4a). The number of these $\mathrm{Dclk1} 1^{+} \mathrm{Cox} 1^{+}$cells was significantly increased in the distal colons of DKO vs. control mice (Fig. 4b). Dclk $1^{+}$cells were absent in DKO;Dclk $1^{\Delta I E C}$ mice, confirming knockout status (Fig. 4a, b); however Cox $1^{+}$ epithelial cells still increased in number in DKO;Dclkl $1^{\Delta I E C}$ vs. control mice showing tuft cells to be intact (Fig. 4c). Dual labeling of DKO;Dclk1 ${ }^{\triangle I E C}$ tissues with the epithelial marker E-cadherin or hematopoietic cell marker CD45 demonstrated that the Cox $1^{+}$cells in DKO;Dclk1 $1^{\Delta I E C}$ crypts were epithelial cell-derived (E-cadherin ${ }^{+}$and CD45') (Figure S5A and B). Further immunostaining with Cox1 and Dclk1 on colonoid sections showed that tuft cell populations were readily found within colonoids from all lines, showing these cells to exist ex vivo in short term (i.e., 1 week) organoid cultures (Figure S5C).

Tuft cells are normally quiescent but can be induced to proliferate in response to inflammatory stimuli [16, 17]. Consistent with this, we found a very low population of proliferating Dclk1-expressing cells in distal colons of control mice, demonstrated by co-staining of Dclk1 with PCNA on colonic sections. As expected, the numbers of $\mathrm{PCNA}^{+}$cells in DKO mice were increased vs. controls; however we also observed increased frequency of dual

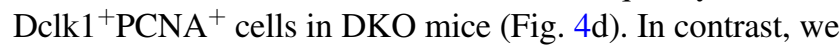
noted the tuft cell population in DKO;Dclk1 ${ }^{\Delta I E C}$ mice did not show a similar increase in proliferative status (Fig. 4d). Of note, Tuft cell $\left(\mathrm{Cox} 1^{+}\right)$status in $D c l k 1^{\Delta I E C}$ mice was similar to controls with respect to number and proliferative status, consistent with the lack of overt disease in the absence of epithelial Dclk1 alone (not shown). Collectively, these studies show that the role of Dclk1 in regulating epithelial repair responses is associated with tuft cell activation status but not necessarily their expansion.

\section{Dclk1-dependent restitution of the surface epithelium during chronic colitis restricts microbial exposure to the lamina propria following mucus breach}

We next determined if the proliferation defect was maintained over the long term during chronic, unremitting disease by examining DKO and DKO;Dclk $1^{\Delta I E C}$ mice at ages 3 

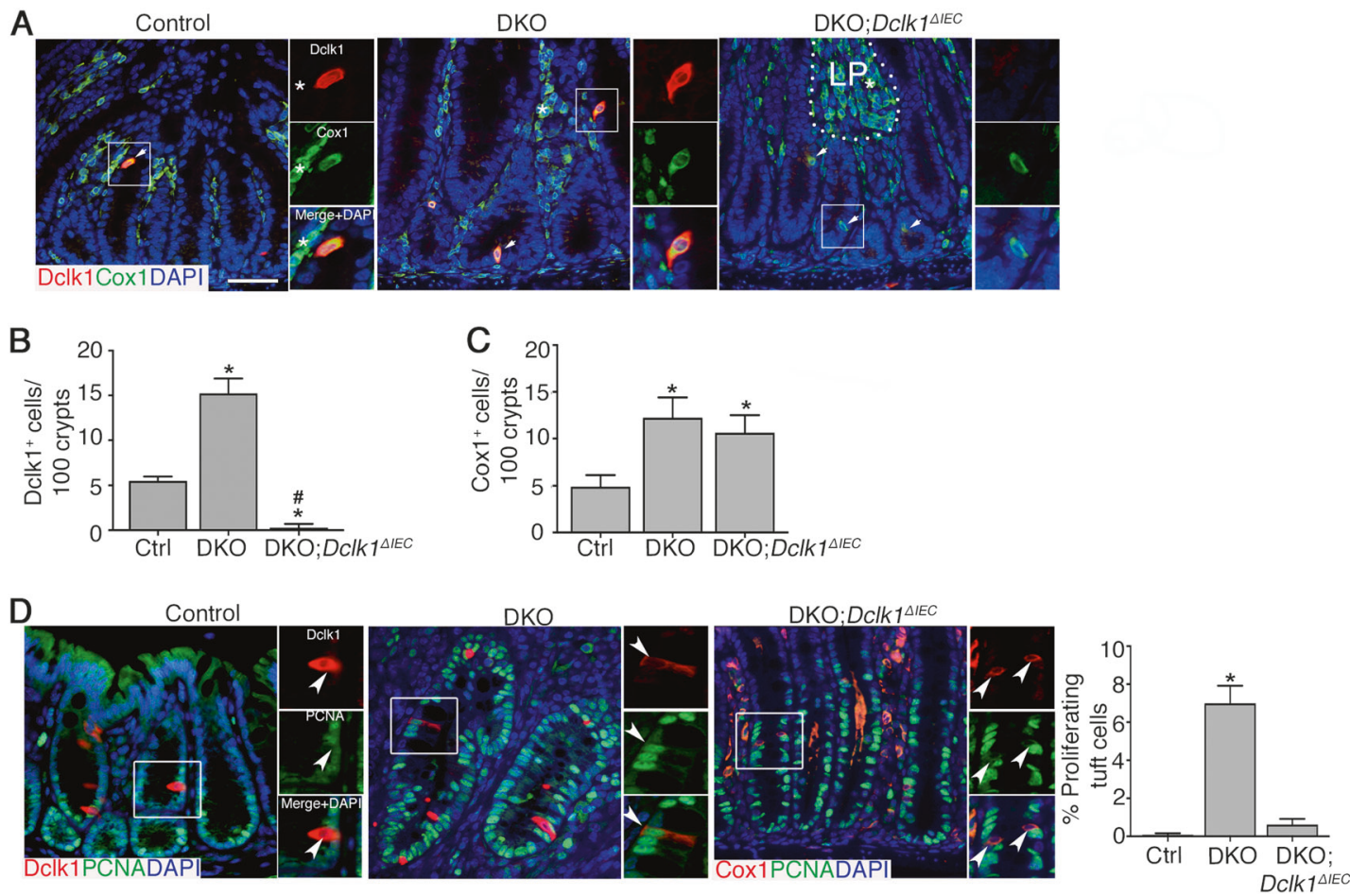

Fig. 4 Dclk1 influences tuft cell activation but not expansion during spontaneous chronic colitis. a Dual staining of Cox 1 and Dclk1 of murine colon tissue. Split images are magnifications of boxed region in corresponding image on left (white). Dashed line, border between crypts and lamina propria (LP). White asterisk, stromal (non-epithelial) Cox $1^{+}$cell. b, $\mathbf{c}$ Quantification of $\operatorname{Dclk} 1^{+}(\mathbf{b})$ and $\operatorname{Cox} 1^{+}(\mathbf{c})$ cells per

and 6.5 months. Analysis of proliferation by Ki67 staining revealed a significant reduction in the number of proliferating cells in distal colon tissues of DKO;Dclk1 $1^{\Delta I E C}$ vs. DKO mice at both timepoints (Fig. 5a). The phenotype was characterized by reduced expansion of the proliferative zone into the upper half of the colonic crypts of DKO;Dclk $1^{\Delta I E C}$ vs. DKO mice (Fig. 5a). We next investigated how defective proliferation exacerbated colitis. We noted the surface mucosa frequently contained fewer epithelial cells vs. DKO, control and $D c l k l^{\Delta I E C}$ mice, leaving the underlying lamina propria exposed to the lumen (Fig. 5b). To determine if increased epithelial cell apoptosis contributes to surface epithelial erosion, we used the terminal deoxynucleotidyl transferase dUTP nick-end labeling (TUNEL) assay, which identifies cell death-induced DNA fragmentation in situ, in combination with E-cadherin staining. Few E-cadherin ${ }^{+}$TUNEL $^{+}$cells were observed within crypts, and no difference was seen among groups (Fig. 5c). These results suggest the proliferation defect contributes to mucosal epithelial erosion by inhibiting restitution of the surface epithelium. We hypothesized the loss of surface epithelium in DKO;Dclk $I^{\Delta I E C}$ mice should contribute to colitis by causing more direct exposure of the lamina propria to colonic bacteria. To support this, confocal analysis of host-bacterial
100 colon crypts. d Dual staining and quantitation of proliferating $\left(\mathrm{PCNA}^{+}\right)$tuft cells $\left(\mathrm{Dclk} 1^{+}\right.$or Cox $\left.1^{+}\right)$in FFPE sections. Split images are magnifications of boxed region (white). Arrowheads, Tuft cell. Results are representative of 7 mice/group, $\sim 3$ weeks of age. For (b): $* P<0.05$ vs. Ctrl; ${ }^{\#} P<0.05$ vs. DKO. For $(\mathbf{c}): * P<0.05$ vs. Ctrl. For (d): $* P<0.05$ vs. all other groups

interactions in these tissues via staining with the EUB338 probe confirmed colonic bacteria could be seen associated with the rectal surface mucosa of both DKO and DKO; $D c l k 1^{\Delta I E C}$ mice whereas bacteria, as expected, were absent from control and $D c l k 1^{\Delta I E C}$ mucosa (Fig. 5d). Taken together these results indicate Dclk1 expression in epithelium leads to long-term impairment of the epithelial regenerative response, which compromises the surface epithelial barrier capacity leading to unrestrained bacterial interactions with lamina propria cells, likely exacerbating inflammation.

\section{Discussion}

Our results demonstrate an important role for epithelial Dclk1, a tuft cell marker, in regulating regenerative responses in chronic colitis, which is required for maintaining epithelial barrier function during bacterial-induced inflammation following mucus destabilization. We found Dclk1 expression in colonic epithelium was restricted to cells that have established tuft cell morphology, suggesting the phenotype was due to defective tuft cell function. Further, the absence of Dclk1 impairs epithelial regenerative 

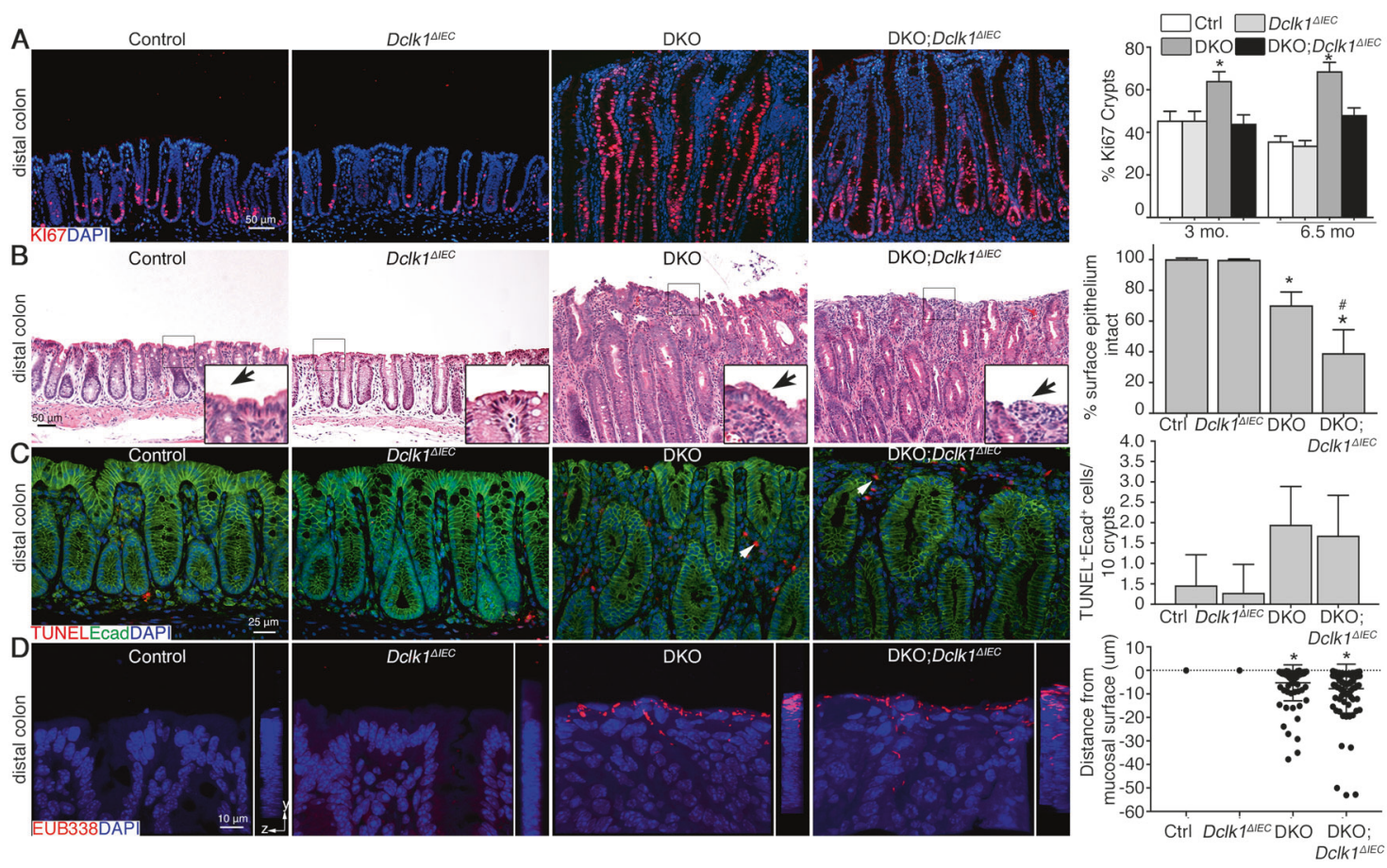

Fig. 5 Dclk1-dependent regeneration contributes to restitution of the surface epithelial barrier to restrict bacterial invasion in the lamina propria. a IF on colon tissue from 6.5-month-old mice. Graph: Quantification of Ki67 at indicated timepoints. b Representative histology shows surface epithelium in distal colon tissue from 3-monthold mice. Inset, magnification of boxed region in corresponding image. Arrows, surface epithelium. Graph: Proportion of mucosa containing surface epithelium. c Dual IF/TUNEL assay on FFPE distal colon sections of 3-month-old mice. Arrows, $\mathrm{TUNEL}^{+}$cell in lamina propria. d Confocal imaging of FISH probe for universal bacteria in colon tissue from normal and inflamed mice. Graph: Quantitation of penetration depth of bacteria into mucosal tissues. Data are representative

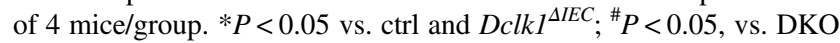

responses induced by microbiota-dependent chronic inflammation, likely through its effects on PGE2 production. Ultimately, Dclk1-dependent restitution of the surface epithelium restricts microbial exposure to the lamina propria following a breach of the mucus layer. Collectively, these studies indicate that epithelial Dclk1 is an important regulator of epithelial function during chronic injury.

Our studies link intestinal epithelial Dclk1 to inflammation-induced proliferative responses. Our data show that despite the chronic colitis, epithelial repair was defective in the absence of epithelial Dclk1, suggesting that Dclk1 expression is necessary to couple the inflammatory response to the regenerative potential of the epithelium. It is noteworthy that, in our model, Caspase (Casp)1-dependent inflammasomes mediate colitis and colitis-induced regeneration [28]. In addition, IL1 $\beta$, a canonical Casp1 substrate, can induce tuft cell activation [16]; therefore, it is plausible IL1 $\beta$ signaling in tuft cells affects pro-regenerative effects of Dclk1, although further studies are needed to confirm this.

Mechanistically, our results link Dclk1 expression to PGE2 production of the inflamed gut. This was likely due to the reduction of Cox 2 gene expression observed in the absence of Dclk1. This is in agreement with previous studies showing Dclk1 signaling in the small intestine promotes PGE2 production during radiation-induced injury [45]. Importantly, we found that stimulation of DKO; $D c l k 1^{\triangle I E C}$ organoids rescued their growth defects to near DKO levels, suggesting PGE2 is a downstream mediator of Dclk1 activity; however, whether this is the case in vivo remains to be studied. This is in contrast to recent studies showing that PGE2 can inhibit intestinal organoid proliferation, and promote differentiation to specialized woundassociated epithelial cells [31]. This discrepancy is likely due to the use of small intestinal organoids in a Wntdepleted media in contrast to our use of colonic organoids in a Wnt-rich media, and may suggest Dclk1 relies on PGE2 to enhance exogenous Wnt signaling to promote optimal proliferation. In this regard, our findings are consistent with previous studies showing PGE2 can increase Wnt signaling pathway activity in adult stem cells by stabilizing $\beta$-catenin via a Protein Kinase A-dependent mechanism [49]. How Dclk1 influences Cox 2 expression and PGE2 production is unclear, especially since Dclk $1^{+}$cells are not prolific the distal colon, even during inflammation. However, a local signal derived from Dclk $1^{+}$tuft cells could be propagated and amplified via associations with enteric neurons [50]. Alternatively, Dclk $1^{+}$tuft cells can intimately interact with 
adjacent epithelial cells through specialized tubules that can access neighboring cell organelles, conceivably leading to consequences on gene expression of adjacent cells [51]. Both scenarios are beyond the scope of the current study, but their relevance to delineating the full spectrum of tuft cell functions in disease warrants their future study. Of note, inhibition of Dclk1 kinase function via the inhibitor LRRK2-in-1 can reduce the proliferative capacity of transformed colorectal and pancreatic cancer lines, suggesting kinase activity of Dclk1 is central to tuft cell function [52].

A consequence of the regenerative defect caused by Dclk1 loss was impaired restitution of the surface epithelium. Interestingly, for reasons that are still unclear, this was most evident in aged mice (i.e., $\geq 3$ months), but was not associated with increased cell death of hypoproliferative epithelium. However, we cannot rule out that heightened cell death of the surface epithelium ultimately contributes its erosion, which would necessitate a robust regenerative response to replace it. Future studies will need to address the interplay between cell death and regeneration, particularly with respect to Dclk1 function, in chronic colitis. Ultimately, the surface erosion correlated with worsened disease as presumably more bacteria would be in direct contact with lamina propria cells. Our results suggest that, although mucosal barrier dysfunction is an intrinsic factor in colitis, maintenance of the surface epithelium limits the severity of the barrier defect and disease outcome. However, since Dclk1 function itself is not sufficient to protect from spontaneous colitis, other barrier functions of the epithelium, especially production of mucus by goblet cells, play more critical roles in maintaining colonic homeostasis.

The function of Dclk1 in our colitis model was dependent upon bacteria-induced inflammation, as epithelialspecific deletion of Dclk1 did not have measurable defects on morphology or physiology of the gut at baseline, similar to a previous study [25]. Inflammation-induced expansion and activation status of tuft cells in our study is consistent with other models of chronic inflammation, such as gastritis [53], and mice infected with Helicobacter hepaticus and Bacteroides fragilis [16], underscoring the role of Dclk1 in inducing tuft cell expansion during bacterial inflammation. However, the activation (i.e., proliferation) status of tuft cells, which are normally highly quiescent under physiologic conditions, was significantly higher in DKO vs. DKO; $D_{c l k 1} I^{\Delta I E C}$ mice. These findings suggest that Dclk1 is important to bring tuft cells out of quiescence, a necessary action for tuft cells to produce progeny [16]. Whether this activation leads to progeny derived from Dclk $1^{+}$cells is unclear. Standard lineage tracing strategies from Dclk $1^{+}$ populations would definitively answer this; however, we are unable to address this in vivo due to the reliance of lineage tracing and our colitis phenotype on the Cre-Lox platform [27]. Nevertheless, ablation of tuft cells or Dclkl in mice demonstrates a similar epithelial proliferation defect upon intestinal insult [16, 24, 25], suggesting some aspects of tuft cell responses are likely through tuft-cell intrinsic Dclk1 signaling.

In conclusion, our studies establish an important protective role of Dclk1 in chronic colitis. By promoting epithelial repair, Dclk1 signing in tuft cells mitigates mucosal barrier dysfunction by replacing cells likely damaged from the chronic inflammation. Of note, Dclk1 functions in a similar way to promote tumorigenesis in mouse models of sporadic cancer (i.e., $A p c^{\mathrm{min} /+}$ mice) [42]. These studies suggest Dclk1 has protective and pathological functions depending on the context of disease, an important consideration in the design of targeted therapies against this kinase.

Acknowledgements This work is supported by grants from National Institutes of Health (R01DK085691, GM114731, and HL128390), Oklahoma Center for the Advancement of Science and Technology (HR13-160), Oklahoma Center for Adult Stem Cell Research, a program of TSET, and National Natural Science Foundation of China (81570504, 81770584, and 31400692).

Author contributions JY, KB, XL, LX conceived of and designed the study; JY, KB, JF, XS, JMM, SM, DQ, CWH acquired the data; JY, $\mathrm{KB}, \mathrm{XL}, \mathrm{LX}$ analyzed and interpreted the data; JY, KB, LX drafted the manuscript.

\section{Compliance with ethical standards}

Conflict of interest The authors declare that they have no conflict of interest.

\section{References}

1. Ford AC, Moayyedi P, Hanauer SB. Ulcerative colitis. BMJ. 2013;346:f432.

2. Abraham C, Cho JH. Inflammatory bowel disease. N Engl J Med. 2009;361:2066-78.

3. Strober W, Fuss I, Mannon P. The fundamental basis of inflammatory bowel disease. J Clin Invest. 2007;117:514-21.

4. Garrett WS, Gallini CA, Yatsunenko T, Michaud M, DuBois A, Delaney ML, et al. Enterobacteriaceae act in concert with the gut microbiota to induce spontaneous and maternally transmitted colitis. Cell Host Microbe. 2010;8:292-300.

5. Darwich AS, Aslam U, Ashcroft DM, Rostami-Hodjegan A. Metaanalysis of the turnover of intestinal epithelia in preclinical animal species and humans. Drug Metab Dispos. 2014;42:2016-22.

6. Clevers H. The intestinal crypt, a prototype stem cell compartment. Cell. 2013;154:274-84.

7. Barker N, van de Wetering M, Clevers $H$. The intestinal stem cell. Genes Dev. 2008;22:1856-64.

8. Barker N, van Es JH, Kuipers J, Kujala P, van den Born M, Cozijnsen $\mathrm{M}$, et al. Identification of stem cells in small intestine and colon by marker gene Lgr5. Nature. 2007;449:1003-7.

9. Barker N, Clevers H. Leucine-rich repeat-containing G-proteincoupled receptors as markers of adult stem cells. Gastroenterology. 2010;138:1681-96.

10. Barker N, van Es JH, Jaks V, Kasper M, Snippert H, Toftgard R, et al. Very long-term self-renewal of small intestine, colon, and 
hair follicles from cycling Lgr5+ve stem cells. Cold Spring Harb Symp Quant Biol. 2008;73:351-6.

11. Sato T, Vries RG, Snippert HJ, van de Wetering M, Barker N, Stange DE, et al. Single Lgr5 stem cells build crypt-villus structures in vitro without a mesenchymal niche. Nature. 2009;459:262-5.

12. Sato T, Stange DE, Ferrante M, Vries RG, Van Es JH, Van den Brink S, et al. Long-term expansion of epithelial organoids from human colon, adenoma, adenocarcinoma, and Barrett's epithelium. Gastroenterology. 2011;141:1762-72.

13. Tian H, Biehs B, Warming S, Leong KG, Rangell L, Klein OD, et al. A reserve stem cell population in small intestine renders Lgr5-positive cells dispensable. Nature. 2011;478:255-9.

14. van Es JH, Sato T, van de Wetering M, Lyubimova A, Nee AN, Gregorieff A, et al. Dll1+ secretory progenitor cells revert to stem cells upon crypt damage. Nat Cell Biol. 2012;14:1099-104.

15. Takeda N, Jain R, LeBoeuf MR, Wang Q, Lu MM, Epstein JA. Interconversion between intestinal stem cell populations in distinct niches. Science. 2011;334:1420-4.

16. Westphalen CB, Asfaha S, Hayakawa Y, Takemoto Y, Lukin DJ, Nuber AH, et al. Long-lived intestinal tuft cells serve as colon cancer-initiating cells. J Clin Invest. 2014;124:1283-95.

17. Middelhoff M, Westphalen CB, Hayakawa Y, Yan KS, Gershon MD, Wang TC, et al. Dclk1-expressing tuft cells: critical modulators of the intestinal niche? Am J Physiol Gastrointest Liver Physiol. 2017;313:G285-99.

18. Gerbe F, Legraverend C, Jay P. The intestinal epithelium tuft cells: specification and function. Cell Mol Life Sci. 2012;69:2907-17.

19. Gerbe F, Sidot E, Smyth DJ, Ohmoto M, Matsumoto I, Dardalhon $\mathrm{V}$, et al. Intestinal epithelial tuft cells initiate type 2 mucosal immunity to helminth parasites. Nature. 2016;529:226-30.

20. Grencis RK, Worthington JJ. Tuft cells: a new flavor in innate epithelial immunity. Trends Parasitol. 2016;32:583-5.

21. Howitt MR, Lavoie S, Michaud M, Blum AM, Tran SV, Weinstock JV, et al. Tuft cells, taste-chemosensory cells, orchestrate parasite type 2 immunity in the gut. Science. 2016;351:1329-33.

22. von Moltke J, Ji M, Liang HE, Locksley RM. Tuft-cell-derived IL-25 regulates an intestinal ILC2-epithelial response circuit. Nature. 2016;529:221-5.

23. Gerbe F, Jay P. Intestinal tuft cells: epithelial sentinels linking luminal cues to the immune system. Mucosal Immunol. 2016;9:1353-9.

24. May R, Qu D, Weygant N, Chandrakesan P, Ali N, Lightfoot SA, et al. Brief report: Dclk1 deletion in tuft cells results in impaired epithelial repair after radiation injury. Stem Cells. 2014;32:822-7.

25. Qu D, Weygant N, May R, Chandrakesan P, Madhoun M, Ali N, et al. Ablation of doublecortin-like kinase 1 in the colonic epithelium exacerbates dextran sulfate sodium-induced colitis. PLoS One. 2015;10:e 0134212.

26. Bergstrom K, Fu J, Johansson ME, Liu X, Gao N, Wu Q, et al. Core 1- and 3-derived O-glycans collectively maintain the colonic mucus barrier and protect against spontaneous colitis in mice. Mucosal Immunol. 2016;10:91-103.

27. Fu J, Wei B, Wen T, Johansson MEV, Liu X, Bradford E, et al. Loss of intestinal core 1-derived O-glycans causes spontaneous colitis in mice. J Clin Invest. 2011;121:1657-66.

28. Bergstrom K, Liu X, Zhao Y, Gao N, Wu Q, Song K, et al. Defective intestinal mucin-type O-glycosylation causes spontaneous colitis-associated cancer in mice. Gastroenterology. 2016;151:152-64.

29. Abramoff MD, Magelhaes PJ, Ram SJ. Image processing with ImageJ. Biophotonics Int. 2004;11:36-42.

30. Miyoshi H, Stappenbeck TS. In vitro expansion and genetic modification of gastrointestinal stem cells in spheroid culture. Nat Protoc. 2013;8:2471-82.
31. Miyoshi H, VanDussen KL, Malvin NP, Ryu SH, Wang Y, Sonnek NM, et al. Prostaglandin E2 promotes intestinal repair through an adaptive cellular response of the epithelium. EMBO J. 2017;36:5-24.

32. Fernando EH, Dicay M, Stahl M, Gordon MH, Vegso A, Baggio $\mathrm{C}$, et al. A simple, cost-effective method for generating murine colonic 3D enteroids and 2D monolayers for studies of primary epithelial cell function. Am J Physiol Gastrointest Liver Physiol. 2017;313:G467-75.

33. Bergstrom KSB, Xia L. Mucin-type O-glycans and their roles in intestinal homeostasis. Glycobiology. 2013;23:1026-37.

34. Bergstrom KS, Guttman JA, Rumi M, Ma C, Bouzari S, Khan MA, et al. Modulation of intestinal goblet cell function during infection by an attaching and effacing bacterial pathogen. Infect Immun. 2008;76:796-811.

35. van Es JH, van Gijn ME, Riccio O, van den Born M, Vooijs M, Begthel $\mathrm{H}$, et al. Notch/[gamma]-secretase inhibition turns proliferative cells in intestinal crypts and adenomas into goblet cells. Nature. 2005;435:959-63.

36. Zheng X, Tsuchiya K, Okamoto R, Iwasaki M, Kano Y, Sakamoto N, et al. Suppression of hath1 gene expression directly regulated by hes 1 via notch signaling is associated with goblet cell depletion in ulcerative colitis. Inflamm Bowel Dis. 2011;17: 2251-60.

37. Yang Q, Bermingham NA, Finegold MJ, Zoghbi HY. Requirement of Math1 for secretory cell lineage commitment in the mouse intestine. Science. 2001;294:2155-8.

38. Katz JP, Perreault N, Goldstein BG, Lee CS, Labosky PA, Yang $\mathrm{VW}$, et al. The zinc-finger transcription factor Klf4 is required for terminal differentiation of goblet cells in the colon. Development. 2002;129:2619-28.

39. Zheng H, Pritchard DM, Yang X, Bennett E, Liu G, Liu C, et al. KLF4 gene expression is inhibited by the notch signaling pathway that controls goblet cell differentiation in mouse gastrointestinal tract. Am J Physiol Gastrointest Liver Physiol. 2009;296:G490-8.

40. Kuhn KA, Manieri NA, Liu TC, Stappenbeck TS. IL-6 stimulates intestinal epithelial proliferation and repair after injury. PLoS One. 2014;9:e114195.

41. Pickert G, Neufert C, Leppkes M, Zheng Y, Wittkopf N, Warntjen $\mathrm{M}$, et al. STAT3 links IL-22 signaling in intestinal epithelial cells to mucosal wound healing. J Exp Med. 2009;206:1465-72.

42. Chandrakesan P, Weygant N, May R, Qu D, Chinthalapally HR, Sureban SM, et al. DCLK1 facilitates intestinal tumor growth via enhancing pluripotency and epithelial mesenchymal transition. Oncotarget. 2014;5:9269-80.

43. Merker SR, Weitz J, Stange DE. Gastrointestinal organoids: how they gut it out. Dev Biol. 2016;420:239-50.

44. Sato T, Clevers H. Growing self-organizing mini-guts from a single intestinal stem cell: mechanism and applications. Science. 2013;340:1190-4.

45. Chandrakesan P, May R, Weygant N, Qu D, Berry WL, Sureban $\mathrm{SM}$, et al. Intestinal tuft cells regulate the ATM mediated DNA damage response via Dclk1 dependent mechanism for crypt restitution following radiation injury. Sci Rep. 2016;6:37667.

46. Stenson WF. Prostaglandins and epithelial response to injury. Curr Opin Gastroenterol. 2007;23:107-10.

47. Pull SL, Doherty JM, Mills JC, Gordon JI, Stappenbeck TS. Activated macrophages are an adaptive element of the colonic epithelial progenitor niche necessary for regenerative responses to injury. Proc Natl Acad Sci USA. 2005;102:99-104.

48. Fukata M, Chen A, Klepper A, Krishnareddy S, Vamadevan AS, Thomas LS, et al. Cox-2 is regulated by Toll-like receptor-4 (TLR4) signaling: role in proliferation and apoptosis in the intestine. Gastroenterology. 2006;131:862-77.

49. Goessling W, North TE, Loewer S, Lord AM, Lee S, StoickCooper CL, et al. Genetic interaction of PGE2 and Wnt signaling 
regulates developmental specification of stem cells and regeneration. Cell. 2009;136:1136-47.

50. Cheng X, Voss U, Ekblad E. Tuft cells: distribution and connections with nerves and endocrine cells in mouse intestine. Exp Cell Res. 2018;369:105-11.

51. Hoover B, Baena V, Kaelberer MM, Getaneh F, Chinchilla S, Bohorquez DV. The intestinal tuft cell nanostructure in 3D. Sci Rep. 2017;7:1652.
52. Weygant N, Qu D, Berry WL, May R, Chandrakesan P, Owen DB, et al. Small molecule kinase inhibitor LRRK2-IN-1 demonstrates potent activity against colorectal and pancreatic cancer through inhibition of doublecortin-like kinase 1. Mol Cancer. 2014;13:103.

53. Tu SP, Quante M, Bhagat G, Takaishi S, Cui G, Yang XD, et al. IFN-gamma inhibits gastric carcinogenesis by inducing epithelial cell autophagy and T-cell apoptosis. Cancer Res. 2011;71:4247-59. 